
\title{
25 Research Soure \\ Burdens of Post-acute Sequelae of COVID-19 by Age, Race, Sex, and Health Status
}

\section{Ziyad Al-Aly ( $\nabla$ zalaly@gmail.com )}

Washington University School of Medicine https://orcid.org/0000-0002-2600-0434

Yan Xie

VA Saint Louis Health Care System https://orcid.org/0000-0002-2457-9382

\section{Benjamin Bowe}

VA Saint Louis Health Care System

\section{Article}

Keywords: Population Groups, Baseline Health, Care Setting, Capacity Planning, Care Strategies

Posted Date: July 12th, 2021

DOI: https://doi.org/10.21203/rs.3.rs-438235/v1

License: (c) (1) This work is licensed under a Creative Commons Attribution 4.0 International License.

Read Full License

Version of Record: A version of this preprint was published at Nature Communications on November 12th, 2021. See the published version at https://doi.org/10.1038/s41467-021-26513-3. 


\section{Abstract}

The Post-Acute Sequelae of SARS-CoV-2 infection (PASC) have been characterized; however, the burden of PASC remains unknown. And whether the burden of individual sequela varies in different population groups is also not clear. Here we estimate that PASC - defined as the presence of at least one sequela in excess of non-infected controls - was $73.43(72.10,74.72)$ per 1000 persons at 6 months. The burden of PASC was 44.51 (43.09, 45.85), $217.08(212.43,222.23)$, and $360.16(350.53,369.38)$ among nonhospitalized, hospitalized, and those who required intensive care during the first 30-days of infection. Burdens of some sequelae were more pronounced in younger individuals, and some were more pronounced in older adults; the same picture was evident in analyses across race, and sex groups. The burden of individual sequela was consistently higher in people with poorer baseline health and increased in a graded fashion according to care setting of the acute infection. In sum, the burden of PASC is substantial; however, PASC is non-monolithic with sequelae that are differentially expressed in various population groups. Collectively, our results may be useful in informing health systems capacity planning and care strategies of people with PASC.

\section{Introduction}

Emerging reports suggest that beyond the acute illness, some COVID-19 survivors experience myriad clinical abnormalities lasting well beyond the first 30 days of infection ${ }^{1-6}$. We recently developed a high dimensional approach to comprehensively and systematically characterize the Post-Acute Sequelae of SARS-CoV-2 infection (PASC) - also referred to as post COVID-19 syndrome, or simply long COVID ${ }^{1}$. Our approach identified 33 sequelae in the pulmonary and several extrapulmonary organ systems including nervous system and neurocognitive disorders, mental health disorders, metabolic disorders, cardiovascular disorders, gastrointestinal disorders, and several other clinical manifestations ${ }^{1}$.

However, significant knowledge gaps remain. Specifically, what is the burden of PASC - defined as having at least one post-acute sequela? And does the burden of individual sequelae differ by age, race, sex, and baseline health status? Addressing these questions has been highlighted as an urgent research priority by stakeholders including the World Health Organization, the United States National Institute of Health, the United Kingdom National Institute for Health and Care Excellence, and several others $2,7-12$. Addressing these questions will help inform capacity planning and care of people with PASC.

Here, we answer this call for urgent research, where we leverage the breadth and depth of the US Department of Veterans Affairs (VA) electronic healthcare databases - which operates the largest integrated health care delivery system in the US - to undertake comprehensive large-scale analyses of 181,384 people with COVID-19 who survived the first 30 days of infection and 4,397,509 non-infected controls, and aimed to estimate the burden of PASC in the overall cohort and among non-hospitalized ( $\mathrm{n}$ $=155,987)$, hospitalized $(n=19,359)$, and those admitted to intensive care $(n=6038)$ and $b)$ burdens of the 33 individual sequelae that comprise PASC - identified in prior work ${ }^{1}$ - in population groups (by age, race, sex, baseline health status). 


\section{Results}

We enrolled 181,384 veterans who survived the first 30 days of COVID infection including 155,987, 19,359 , and 6038 who were non-hospitalized, hospitalized, and admitted to intensive care during the acute phase (the first 30 days) of the COVID-19 infection. The control group included 4,397,509 users of the Veterans Health administration (VHA) with no known COVID-19 infection. The baseline demographic and health characteristics are described in Supplementary Table 1. The unadjusted number and percentage of incident clinical manifestations in the control group (VHA users), in the overall COVID-19 cohort, and according to COVID-19 care setting (non-hospitalized, hospitalized, and admitted to intensive care) during the follow up is presented in Supplementary Table 2.

In adjusted analyses, 30-day survivors of COVID-19 exhibited increased risk of a broad range of incident sequelae including pulmonary system disorders and cardiovascular, coagulation, dermatologic, endocrine, gastrointestinal, kidney, mental health, musculoskeletal, and neurologic system disorders; risks and associated burdens for each sequela are provided in Supplementary Fig. 1, Supplementary Table 3.

\section{Burden of PASC in overall cohort according to care setting of the acute infection (non-hospitalized, hospitalized, and required intensive care):}

To gain a better understanding of the toll of long covid in 30-day survivors of acute COVID-19, we first estimated the burden of PASC - defined as having at least one sequela in excess of the control group (VHA users without COVID-19). The burden of PASC in the overall cohort was $73,43(72.10,74.72)$ per 1000 persons at 6 months (Table 1), and increased according to care setting of the acute infection and was $44.51(43.09,45.85), 217.08(212.43,222.23)$, and $360.16(350.53,369.38)$ per 1000 persons at 6 months among non-hospitalized, hospitalized, and those who required intensive care during the first 30days of infection, respectively) (Table 1 and Fig. 1 ). 
Table 1

Overall burden of Post-Acute Sequelae of SARS-CoV-2 infection (PASC) per 1000 persons at 6 months in the overall cohort and across care setting (non-hospitalized, hospitalized, and admitted to intensive care during the acute phase of the infection).

\begin{tabular}{|c|c|c|c|}
\hline & \multirow{2}{*}{$\begin{array}{l}\text { COVID-19 group } \\
\text { Adjusted burden per } 1000 \\
\text { persons at } 6 \text { months ( } 95 \% \\
\text { Cl) }\end{array}$} & \multirow{2}{*}{$\begin{array}{l}\text { Users of the VHA without } \\
\text { COVID- } 19 \\
\text { Adjusted burden per } 1000 \\
\text { persons at } 6 \text { months ( } 95 \% \\
\text { Cl) }\end{array}$} & \multirow{2}{*}{$\begin{array}{l}\text { Burden associated with } \\
\text { COVID-19 } \\
\text { Adjusted burden per } 1000 \\
\text { persons at } 6 \text { months ( } 95 \% \\
\text { Cl) }\end{array}$} \\
\hline & & & \\
\hline \multirow[t]{2}{*}{ Overall } & 168.01 & 94.56 & 73.43 \\
\hline & $(166.68,169.29)$ & $(94.23,94.88)$ & $(72.1,74.72)$ \\
\hline \multirow{2}{*}{$\begin{array}{l}\text { Non- } \\
\text { hospitalized } \\
\text { COVID-19* }\end{array}$} & 139.07 & 94.56 & 44.51 \\
\hline & $(137.63,140.44)$ & $(94.23,94.88)$ & $(43.09,45.85)$ \\
\hline \multirow{2}{*}{$\begin{array}{l}\text { Hospitalized } \\
\text { COVID-19* }\end{array}$} & 311.61 & 94.56 & 217.08 \\
\hline & $(306.90,316.82)$ & $(94.23,94.88)$ & $(212.43,222.23)$ \\
\hline \multirow{2}{*}{$\begin{array}{l}\text { COVID-19 } \\
\text { required } \\
\text { ICU* }\end{array}$} & 454.74 & 94.56 & 360.16 \\
\hline & $(445.01,463.92)$ & $(94.23,94.88)$ & $(350.53,369.38)$ \\
\hline \multicolumn{4}{|c|}{ *. Care settings during the first 30 days of infection. } \\
\hline \multicolumn{4}{|c|}{$\begin{array}{l}\text { †. Burden defined as having at least one sequela in excess of users of the Veterans Health } \\
\text { Administration without COVID-19 after the first 30-days of infection. Burden was estimated based on } \\
\text { Poisson regression. }\end{array}$} \\
\hline
\end{tabular}

\section{Burden of individual sequelae by age, race, sex, and baseline health status:}

To gain a better understanding of whether and to what extent the burden of each sequalae may be differentially expressed across various population groups, we estimated the burden of individual postacute sequelae per 1000 persons at 6 months (the burden in excess of the control group) by age, race, sex, and baseline health status represented by the number of comorbidities at baseline (Fig. 2 and Supplementary Table 4-5). This analysis showed that the burdens were not uniformly expressed across age, race, sex, and baseline health status. Analyses of differences in burden on the basis of age suggest that while most incident sequelae were higher in older adults; burdens of hyperlipidemia, chest pain, sleep disorders, headache, obesity, mood disorders, cough and smell problems were higher in people younger than 60 years old. Smaller differences of burden on the basis of race were observed where burden of acute kidney injury, diabetes mellitus, chest pain, cough, substance abuse, thromboembolism, headache and tachycardia were slightly higher in Black persons; GERD and smell problems were higher in White persons. Burden of several sequelae in the respiratory system disorders (shortness of breath and cough), cardiovascular system disorders (chest pain, and arrythmias), neurologic system disorders (headache, and smell problems), and dermatologic disorders (hair loss, and skin rash) were higher in females. Nearly 
all sequelae were more pronounced in persons with higher burden of baseline comorbidities (Fig. 3 and Supplementary Table 6).

\section{Burden of sequelae by age, race, sex, and baseline health status according to care setting of the acute infection (non-hospitalized, hospitalized, and required intensive care):}

In the overall cohort, burden of individual sequelae increased according to the care setting of the acute infection from non-hospitalized, hospitalized, and those who required intensive care (Fig. 4a-c,

Supplementary Table 7-9). In each subgroup based on age, race, sex, and baseline health status. the burden for each sequela increased as the intensity of care setting of the acute infection increased (nonhospitalized, hospitalized, admitted to intensive care).

\section{Negative Controls:}

Testing of risks of accidental injuries and neoplasms as negative outcome controls in people with COVID19 (the overall COVID-19 cohort) yielded non-significant association between COVID-19 exposure and the risks of accidental injuries and neoplasms (Hazard ratio: $1.04(0.84,1.29)$ and $1.01(0.88,1.17)$, respectively) (Supplementary Table 10). The examination of negative control in groups according to care setting in non-hospitalized, hospitalized, and admitted to intensive care for COVID-19 also yielded nonsignificant associations - results that were consistent with a priori expectations (Supplementary Table 10).

\section{Discussion}

In this work, we estimate that PASC - defined as at least one sequela in excess of a non-infected control group - was $73.43(72.10,74.72)$ per 1000 persons at 6 months. The burden of PASC increased as a function of the severity of the acute infection as proxied by the care setting and was $44.51(43.09,45.85)$, $217.08(212.43,222.23)$, and $360.16(350.53,369.38)$ per 1000 persons at 6 months among nonhospitalized, hospitalized, and those who required intensive care during the first 30-days of infection, respectively. PASC is a multifaceted non-monolithic entity; some of its sequelae were more pronounced in younger individuals, and some were more pronounced in older adults. The same picture was evident in analyses across race groups (White and Black participants), and sex (males and females). The burden of individual sequela was consistently higher in people with poorer baseline health status and increased in a graded fashion according to intensity of the care setting of the acute infection. The constellation of findings shows that among 30-day survivors of COVID-19, the burden of PASC is substantial (7\%); PASC is not a monolithic entity with sequelae that are differentially expressed in various population groups. Collectively, our results provide estimates that may be useful in informing health systems capacity planning and care strategies of people with PASC.

The implications of our results are clear. As the number of COVID-19 cases continues to climb across the globe, health systems face the dual challenge of coping with surges in acute infections, and caring for COVID-19 survivors (now accounting for more than $2 \%$ of the global population and growing) who will 
also likely require substantial care to mitigate permanent health loss. This will place additional demands on already strained health systems. Governments and health systems around the globe should be actively devising plans to address the tide of COVID-19 survivors in need of post COVID-19 care. Our estimates of the burden of PASC (around 7\% of infected people) and burden of 33 individual sequelae by age, race, sex, and baseline health status may help inform capacity planning and optimal composition of multidisciplinary post COVID-19 clinics $^{10}$.

Estimates of the burden of individual sequela by age, race, sex, and baseline health status suggest a more nuanced picture in that the burden of some sequela was more pronounced in younger adults (e.g. sleep disorders, headache, mood disorders, and smell problems), Black participants (e.g. new onset diabetes mellitus, chest pain, substance abuse, thromboembolism, headache, and tachycardia), females (e.g. chest pain, arrythmias, headache, smell problems, hair loss, and skin rash). These estimates provide insights into the long-term health consequences of COVID-19 and suggest that PASC is a complex nonmonolithic entity that may manifest differently in various population groups.

PASC is a multifaceted entity with broad symptomatology and clinical abnormalities spanning multiple organ system. To date, there is no clear unifying hypothesis for a mechanism that explains the myriad sequelae of post-acute COVID-19. Some of post-acute clinical manifestations may be a direct result of some acute clinical manifestations that morphed into subacute and chronic conditions. It is also possible that some sequelae may be due to the immune response to the initial infection (e.g. through hyperactivated immune response and autoimmunity or persistence of the virus in immune privileged sites $^{13}$. Lessons learned from prior natural disasters and previous pandemics also suggest the putative presence of indirect effects including changes in the broader contextual environment, social (e.g. isolation, quarantine, reduced social contact and loneliness), economic (e.g. financial distress due to complete loss or reduced income), and behavioral conditions (e.g. changes in dietary habits and physical activity), lived experiences of trauma and grief (from pandemic related happenings) that may be differentially experienced by people with COVID-19 may also shape health outcomes in COVID-19 survivors $^{14-19}$. A better understanding of the biologic mechanisms and pathophysiology of PASC will facilitate the development of treatment strategies to lessen the burden of chronic illness and reduce permanent health loss and mortality among people with PASC.

Here we use the term "PASC" to denote the consequences of post-covid in excess of what can be ascertained in the control group; while this definition is epidemiologically useful to estimate burden of disease, a clinical definition is needed to guide diagnosis and treatment ${ }^{20}$. Other terms used in the literature include "Iong COVID-19", "post-COVID-19 syndrome" and "post-acute COVID-19 syndrome", and people with symptoms and clinical manifestations beyond the acute phase have been referred to in the lay vernacular as "long haulers" 3 . We recognize that this matter is subject to intense research by the scientific community and patient groups with lived experiences of post COVID-19 who hold a broad range of views regarding these terminologies ${ }^{12,20-22}$. Identification of scientifically accurate definitions and culturally sensitive terms to describe the illness beyond the acute phase will be an important step not only 
to standardize scientific communications globally but also to support clear and consistent public health messaging about the long-term consequences of COVID- $19^{20}$.

The study has several strengths. We used the vast electronic health databases of the US Department of Veterans Affairs national health care databases - the largest nationally integrated healthcare delivery system in the US - to estimate burden of overall PASC and its individual sequelae by age, race, sex, and baseline health status. We comprehensively examined 33 sequelae which were defined based on integrated data from multiple sources including diagnostic codes, medications, and laboratory test results. The simultaneous examination of incident sequelae in the same analytic framework allows the comparative evaluation of risks and burdens of these conditions - providing health care providers, health system planners, public health officials, and the public at large with a priority list of the post-acute clinical conditions encountered in COVID-19 survivors. For each outcome examined, we built a cohort free of the related outcome at baseline to identify the risk of incident outcome during follow up - this approach allows the identification of incident clinical manifestations and abnormalities following COVID-19 infection. While we conducted survival analyses to estimate the risk of each outcome examined, we - for each outcome - also estimated the excess burden per 1000 persons due to COVID-19; this measure of risk on the absolute scale also considers the baseline risk and provides a more meaningful estimate of potential harm and can be more effectively communicated to the wider public than measures of relative risks (e.g. hazard ratio).

This study has several limitations. Our approach does not provide mechanistic insights into PASC nor does it delineate the sequelae that are direct or indirect consequences of the COVID-19 infection. Because of the predominantly male composition of the VA population, our findings may not identify clinical manifestations of post-acute COVID-19 that may be differentially much more pronounced in females and either non-expressed or rare in males. Finally, COVID-19 patients were enrolled in our cohorts from March 01 to March 15, 2021 and followed until May 01, 2021; as the COVID-19 global pandemic continues to evolve, and as treatment strategies improve, new variants of the virus emerge, and vaccine availability increases, it is likely that the epidemiology, short term, and long term outcomes of COVID-19 will likely also change over time.

In conclusion, we estimate that burden of PASC is around 7\%; while the burden of PASC increased according to the severity of the care setting of the acute COVID-19 infection, it was not trivial (4.4\%) among those who were not hospitalized for acute COVID-19. Our results also show that PASC is not monolithic; the burden of its individual components may be differentially expressed in various population groups. Together, the estimates provided here suggest that the toll of morbidity of COVID-19 is extends well beyond the acute phase. While, optimism is rising that - as vaccine availability increases - the pandemic may soon be behind us, the focus on the immediate health effects of COVID-19 allows visibility of the tip of the iceberg. The long-term consequences of COVID-19 - as evidenced in our work - are substantial and will reverberate for a long time after the surges in acute infections abate. Long COVID (or as we refer to it in this work PASC) is a complex multifaceted non-monolithic post-viral syndrome; it demands greater attention and a coordinated long-term global response strategy. 


\section{Methods}

\section{Setting:}

The study utilized the VA electronic health care databases. The VA provides health care to US Veterans and operates the largest national integrated healthcare system in the United States with 1,255 health care facilities, including 170 VA medical centers and 1,074 outpatient sites located across the United States. Veterans enrolled have access to the Department of Veterans Affairs comprehensive medical benefits package including inpatient hospital care; outpatient services; preventive, primary, and specialty care; prescriptions; mental healthcare; home healthcare; geriatric and extended care; medical equipment; and prosthetics. VA electronic health care databases are update daily.

\section{Cohort:}

US Veterans who encountered the VHA between January 01, 2019 and December 31, 2019 were selected $(\mathrm{N}=5,808,018)$. Among those alive on March 01, $2020(\mathrm{~N}=5,606,309)$, the COVID-19 group consisted of those with a COVID-19 positive test between March 01, 2020 and March 15, 2021 ( $n=98,661)$. We then selected those who were alive on the $30^{\text {th }}$ day after their first positive test $(N=181,384)$. COVID-19 positive patients were separated into 3 groups based on the care received during the 30 days after the positive test: 1) non-hospitalized $(N=155,987) ; 2)$ hospitalized $(N=19,359) ; 3)$ received intensive care $(N=6038)$. To generate a comparison group without COVID-19, we selected 4,534,600 participants who did not have a COVID-19 positive test, and randomly assigned every 25 of them the same cohort enrollment date as one participant in the COVID-19 group. We then further selected from the control group who were alive during the first 30 days after the date of enrollment (VHA group $n=4,397,509$ ). Participants were followed until May 01, 2021 (Supplementary Figure 2).

\section{Data sources:}

Data used in this study was collected from the VA Corporate Data Warehouse (CDW) ${ }^{23-28}$. The CDW Outpatient Encounters and Inpatient Encounters domains provided information related to diagnoses, procedures and hospitalization records ${ }^{29}$. The CDW Outpatient Pharmacy domain and CDW Bar Code Medication Administration domain were used to collect prescription information. CDW Patient domain was used to collect demographic information. The CDW Laboratory Results domain was used to collect laboratory test information, and the COVID-19 Shared Data Resource was used to collect COVID-19 tests. The Area Deprivation Index (ADI), a composite measure of income, education, employment, and housing was obtained from the University of Wisconsin ${ }^{30}$.

\section{Negative outcome controls:}

The use of negative controls in observational studies may help detect the presence of both suspected and unsuspected spurious biases; the application of negative controls will test if shared biases in outcome ascertainment, residual confounding, analytic approach, or other latent biases might have 
influenced the results ${ }^{31,32}$. Here we followed the approach outlined by Lipsitch and collaborators to test accidental injuries and neoplasms as negative outcome controls ${ }^{31}$, where based on current knowledge, we would expect no association between COVID-19 infection and these 2 negative outcome controls.

\section{Post-acute sequelae of COVID-19}

We examined a set of 33 post-acute COVID-19 outcomes; these outcomes were selected based on prior studies $^{1,33}$, review of the literature ${ }^{2,3}$, and the most recent US National Institute of Health workshop on PASC. Outcomes were defined based on ICD10 codes recorded from inpatient or outpatient encounters, medication records, or laboratory tests when appropriate using definitions validated for use with electronic health records ${ }^{33-44}$. Detailed definitions of the outcomes are presented in Supplementary table 11. Cardiovascular outcomes included acute coronary disease, arrythmias, bradycardia, chest pain, heart failure, myocarditis and tachycardia; coagulation outcomes included thromboembolism; dermatologic outcome included hair loss and skin rash; endocrine outcome included diabetes mellitus, hyperlipidemia and obesity; gastrointestinal outcome included constipation, diarrhea and GERD, general outcome include fatigue; kidney outcome include acute kidney injury and chronic kidney disease; mental health outcome included anxiety, depression, mood disorder, sleep disorder and substance abuse; musculoskeletal outcome included joint pain and muscle weakness; neurologic outcome included headache, memory problems, smell problems and stroke; pulmonary outcome included cough, hypoxemia and shortness of breath. Occurrence of incident clinical manifestation was defined as the occurrence of a manifestation that did not occur within past one year before cohort enrollment. PASC was defined as the presence of at least one incident clinical manifestation in excess of the non-infected controls.

\section{Covariates:}

Covariates for analyses included age, race (White, Black, and Other), sex, receipt of long-term care, Area Deprivation Index based on patient addresses and proxies of healthcare utilization such as number of outpatient encounters, number of hospital admissions, number of outpatient prescriptions and number of outpatient serum creatinine measurements in the year before enrollment. We also included comorbidities such as chronic lung disease, cancer, cardiovascular disease, cerebrovascular disease, dementia, diabetes mellitus, hypertension, hyperlipidemia, depression, anxiety, chronic kidney disease, hepatitis C and peripheral artery disease. In addition, covariates included overweight, obesity, smoking status (never, former, and current) and the Charlson comorbidity index were also adjusted for. We also adjusted for US geographic region (West, Mid-west, South and Northeast) where the care was received, and additional health system characteristics including total number of beds, number of COVID-19 tests administered, COVID-19 positivity rate, and average hospital bed occupancy during the week of participant enrollment.

\section{Statistical analyses:}

Characteristics of the VHA users without COVID-19, and those with COVID-19 according to care setting of the acute infection (non-hospitalized, hospitalized, and admitted to intensive care) were described. 
Excess burden of PASC, defined as having at least one sequela in excess of VHA users without COVID-19, was estimated using Poisson regressions, where burden was defined as the number of incident sequelae occurring during follow-up. The excess burdens of having 1, 2, to 33 PASC at 6 months, as well as the total excess number of PASC, were estimated in the overall cohort and by care setting of the acute infection.

We then estimated the excess burden of incident individual sequela. For each outcome examined, we built a cohort of participants without a history of the outcome. Cox models adjusting for covariates were used to estimate the hazard ratio of each COVID-19 care setting compared to VHA users, and the survival probability for the 4 groups at 6 months. Cause specific hazard models were used where occurrence of death was considered as competing risk during the analyses. Excess burden per 1000 patients at 6 months were computed as the difference in survival probability between each COVID-19 care setting and the VHA users. Burden of outcomes in the overall COVID-19 population was computed as the weighted sum of the burden of the three care settings based on the proportion of COVID-19 patients in each care setting. Analyses were also conducted to estimate the excess burden within subgroups by age, race, sex, and baseline health status. Burden differences between subgroups of age $\leq 60$ and $>70$, Black and White, female and male, and 0 and $>3$ comorbidity score were then estimated.

All analyses were done using SAS Enterprise Guide version 7.1 (SAS Institute, Cary, NC). Data visualizations were performed in R 4.0.3 (R Foundation for Statistical Computing, Vienna, Austria). The study was approved by the Institutional Review Board of the Department of Veterans Affairs St. Louis Health Care System, St. Louis, MO.

\section{Declarations}

Data availability: The data that support the findings of this study are available from the US Department of Veterans Affairs.

Code Availability: All SAS and R programing codes will be made available upon request.

Acknowledgements: This study used data from the VA COVID-19 Shared Data Resource.

Author Contributions: ZAA and YX contributed to the development of the study concept and design. ZAA and $Y X$ contributed to data analysis. ZAA and $Y X$ contributed to interpretation of results. ZAA and $Y X$ drafted the manuscript. ZAA, YX, and BB contributed to critical revision of the manuscript. ZAA provided administrative, technical, and material support, as well as supervision and mentorship. Each author contributed important intellectual content during manuscript drafting or revision and accepts accountability for the overall work by ensuring that questions pertaining to the accuracy or integrity of any portion of the work are appropriately investigated and resolved. All authors approved the final version of the report. The corresponding author attests that all the listed authors meet the authorship criteria and that no others meeting the criteria have been omitted. 
Funding: This research was funded by the United States Department of Veterans Affairs and the Institute for Public Health at Washington University in Saint Louis, Missouri, USA (for ZAA), and two American Society of Nephrology and KidneyCure fellowship awards (for BB and YX).

Disclaimer: The contents do not represent the views of the US Department of Veterans Affairs or the US government.

Ethical approval: This research project was reviewed and approved by the Institutional Review Board of the Department of Veterans Affairs Saint Louis Health Care System.

Competing interests: The authors declare no conflict of interest.

\section{References}

1. Al-Aly, Z., Xie, Y. \& Bowe, B. High-dimensional characterization of post-acute sequelae of COVID-19. Nature, doi:10.1038/s41586-021-03553-9 (2021).

2. In the wake of the pandemic: Preparing for Long COVID. World Health Organization (2021).

3. Greenhalgh, T., Knight, M., A'Court, C., Buxton, M. \& Husain, L. Management of post-acute covid-19 in primary care. Bmj 370, m3026, doi:10.1136/bmj.m3026 (2020).

4. Ayoubkhani, D. et al. Post-covid syndrome in individuals admitted to hospital with covid-19: retrospective cohort study. Bmj 372, n693, doi:10.1136/bmj.n693 (2021).

5. Dennis, A. et al. Multiorgan impairment in low-risk individuals with post-COVID-19 syndrome: a prospective, community-based study. BMJ Open 11, e048391, doi:10.1136/bmjopen-2020-048391 (2021).

6. Daugherty, S. E. et al. Risk of clinical sequelae after the acute phase of SARS-CoV-2 infection: retrospective cohort study. Bmj 373, n1098, doi:10.1136/bmj.n1098 (2021).

7. Collins, F. S. in NIH launches new initiative to study "Long COVID" (National Institutes of Health, Wachington, DC, 2021).

8. NHS England. National guidance for post-covid syndrome assessment clinics.. (2020).

9. NICE. Covid-19 rapid guideline: managing the long-term effects of covid-19.. (2020).

10. Sivan, M. \& Taylor, S. NICE guideline on long covid. Bmj 371, m4938, doi:10.1136/bmj.m4938 (2020).

11. Yelin, D. et al. Long-term consequences of COVID-19: research needs. Lancet Infect Dis 20, 11151117, doi:10.1016/S1473-3099(20)30701-5 (2020).

12. The, L. Facing up to long COVID. Lancet 396, 1861, doi:10.1016/S0140-6736(20)32662-3 (2020).

13. Long-term immunological health consequences of COVID-19. British Society for Immunology (2020).

14. Figueroa, J. D. et al. Distinguishing between direct and indirect consequences of covid-19. Bmj 369, m2377, doi:10.1136/bmj.m2377 (2020).

15. Townsend, E. COVID-19 policies in the UK and consequences for mental health. Lancet Psychiatry 7 , 1014-1015, doi:10.1016/S2215-0366(20)30457-0 (2020). 
16. Knipe, D., Evans, H., Marchant, A., Gunnell, D. \& John, A. Mapping population mental health concerns related to COVID-19 and the consequences of physical distancing: a Google trends analysis. Wellcome open research 5, 82, doi:10.12688/wellcomeopenres.15870.2 (2020).

17. Raker, E. J., Zacher, M. \& Lowe, S. R. Lessons from Hurricane Katrina for predicting the indirect health consequences of the COVID-19 pandemic. Proceedings of the National Academy of Sciences of the United States of America 117, 12595-12597, doi:10.1073/pnas.2006706117 (2020).

18. Mahase, E. Covid-19: Mental health consequences of pandemic need urgent research, paper advises. Bmj 369, m1515, doi:10.1136/bmj.m1515 (2020).

19. Xie, Y., Bowe, B., Yan, Y., Cai, M. \& Al-Aly, Z. County-Level Contextual Characteristics and Disparities in Life Expectancy. Mayo Clinic proceedings 96, 92-104, doi:10.1016/j.mayocp.2020.04.043 (2021).

20. Alwan, N. A. \& Johnson, L. Defining long COVID: Going back to the start. Med (N Y), doi:10.1016/j.medj.2021.03.003 (2021).

21. Gorna, R. et al. Long COVID guidelines need to reflect lived experience. Lancet, doi:10.1016/S01406736(20)32705-7 (2020).

22. Davis, H. E. et al. Characterizing Long COVID in an International Cohort: 7 Months of Symptoms and Their Impact. medRxiv, 2020.2012.2024.20248802, doi:10.1101/2020.12.24.20248802 (2020).

23. Xie, Y. et al. Proton Pump Inhibitors and Risk of Incident CKD and Progression to ESRD. Journal of the American Society of Nephrology: JASN 27, 3153-3163, doi:10.1681/ASN.2015121377 (2016).

24. Xie, Y. et al. Risk of death among users of Proton Pump Inhibitors: a longitudinal observational cohort study of United States veterans. BMJ Open 7, e015735, doi:10.1136/bmjopen-2016-015735 (2017).

25. Xie, Y. et al. Long-term kidney outcomes among users of proton pump inhibitors without intervening acute kidney injury. Kidney internationa/91, 1482-1494, doi:10.1016/j.kint.2016.12.021 (2017).

26. Xie, Y. et al. Higher blood urea nitrogen is associated with increased risk of incident diabetes mellitus. Kidney international 93, 741-752, doi:10.1016/j.kint.2017.08.033 (2018).

27. et al. Clinical Implications of Estimated Glomerular Filtration Rate Dip Following Sodium-Glucose Cotransporter-2 Inhibitor Initiation on Cardiovascular and Kidney Outcomes. Journal of the American Heart Association 0, e020237, doi:doi:10.1161/JAHA.120.020237.

28. Cai, M. et al. Temporal Trends in Incidence Rates of Lower Extremity Amputation and Associated Risk Factors Among Patients Using Veterans Health Administration Services From 2008 to 2018. JAMA Netw Open 4, e2033953, doi:10.1001/jamanetworkopen.2020.33953 (2021).

29. Vincent, B. M., Wiitala, W. L., Burns, J. A., Iwashyna, T. J., Prescott, H. C. Using veterans affairs corporate data warehouse to identify 30-day hospital readmissions. Health Services and Outcomes Research Methodology 18, 143-154 (2018).

30. Kind, A. J. \& Buckingham, W. R. Making Neighborhood-Disadvantage Metrics Accessible-The Neighborhood Atlas. New England Journal of Medicine 378, 2456-2458 (2018). 
31. Lipsitch, M., Tchetgen Tchetgen, E. \& Cohen, T. Negative controls: a tool for detecting confounding and bias in observational studies. Epidemiology 21, 383-388, doi:10.1097/EDE.0b013e3181d61eeb (2010).

32. Shi, X., Miao, W. \& Tchetgen, E. T. A Selective Review of Negative Control Methods in Epidemiology. Current epidemiology reports 7, 190-202, doi:10.1007/s40471-020-00243-4 (2020).

33. Xie, Y., Bowe, B., Maddukuri, G. \& Al-Aly, Z. Comparative evaluation of clinical manifestations and risk of death in patients admitted to hospital with covid-19 and seasonal influenza: cohort study. Bmj, doi:http://dx.doi.org/10.1136/bmj.m4677 (2021).

34. Xie, Y. et al. Estimates of all cause mortality and cause specific mortality associated with proton pump inhibitors among US veterans: cohort study. Bmj 365, I1580, doi:10.1136/bmj.I1580 (2019).

35. Xie, Y. et al. Comparative Effectiveness of SGLT2 Inhibitors, GLP-1 Receptor Agonists, DPP-4 Inhibitors, and Sulfonylureas on Risk of Kidney Outcomes: Emulation of a Target Trial Using Health Care Databases. Diabetes care 43, 2859-2869, doi:10.2337/dc20-1890 (2020).

36. Xie, Y. et al. Comparative Effectiveness of the Sodium-Glucose Cotransporter 2 Inhibitor Empagliflozin Versus Other Antihyperglycemics on Risk of Major Adverse Kidney Events. Diabetes care 43, 2785-2795, doi:10.2337/dc20-1231 (2020).

37. Bowe, B. et al. Acute Kidney Injury in a National Cohort of Hospitalized US Veterans with COVID-19. Clinical journal of the American Society of Nephrology: CJASN, doi:10.2215/CJN.09610620 (2020).

38. Bowe, B. et al. The $2016 \mathrm{global}$ and national burden of diabetes mellitus attributable to PM2.5 air pollution. The Lancet. Planetary health 2, e301-e312, doi:10.1016/S2542-5196(18)30140-2 (2018).

39. Bowe, B., Xie, Y., Xian, H., Balasubramanian, S. \& Al-Aly, Z. Low levels of high-density lipoprotein cholesterol increase the risk of incident kidney disease and its progression. Kidney internationa/ $\mathbf{8 9}$, 886-896, doi:10.1016/j.kint.2015.12.034 (2016).

40. Bowe, B. et al. High Density Lipoprotein Cholesterol and the Risk of All-Cause Mortality among U.S. Veterans. Clinical journal of the American Society of Nephrology: CJASN 11, 1784-1793, doi:10.2215/CJN.00730116 (2016).

41. Bowe, B. et al. Ambient Fine Particulate Matter Air Pollution and Risk of Weight Gain and Obesity in United States Veterans: An Observational Cohort Study. Environmental Health Perspectives 129, 047003, doi:doi:10.1289/EHP7944 (2021).

42. Bowe, B. et al. Ambient fine particulate matter air pollution and the risk of hospitalization among COVID-19 positive individuals: Cohort study. Environment International 154, 106564, doi:https://doi.org/10.1016/j.envint.2021.106564 (2021).

43. Xie, Y. et al. Clinical Implications of Estimated Glomerular Filtration Rate Dip Following SodiumGlucose Cotransporter-2 Inhibitor Initiation on Cardiovascular and Kidney Outcomes. Journal of the American Heart Association 10, e020237, doi:10.1161/JAHA.120.020237 (2021).

44. Xie, Y. et al. Comparative Effectiveness of Sodium-Glucose Cotransporter 2 Inhibitors vs Sulfonylureas in Patients With Type 2 Diabetes. JAMA internal medicine, doi:10.1001/jamainternmed.2021.2488 (2021). 
Figures
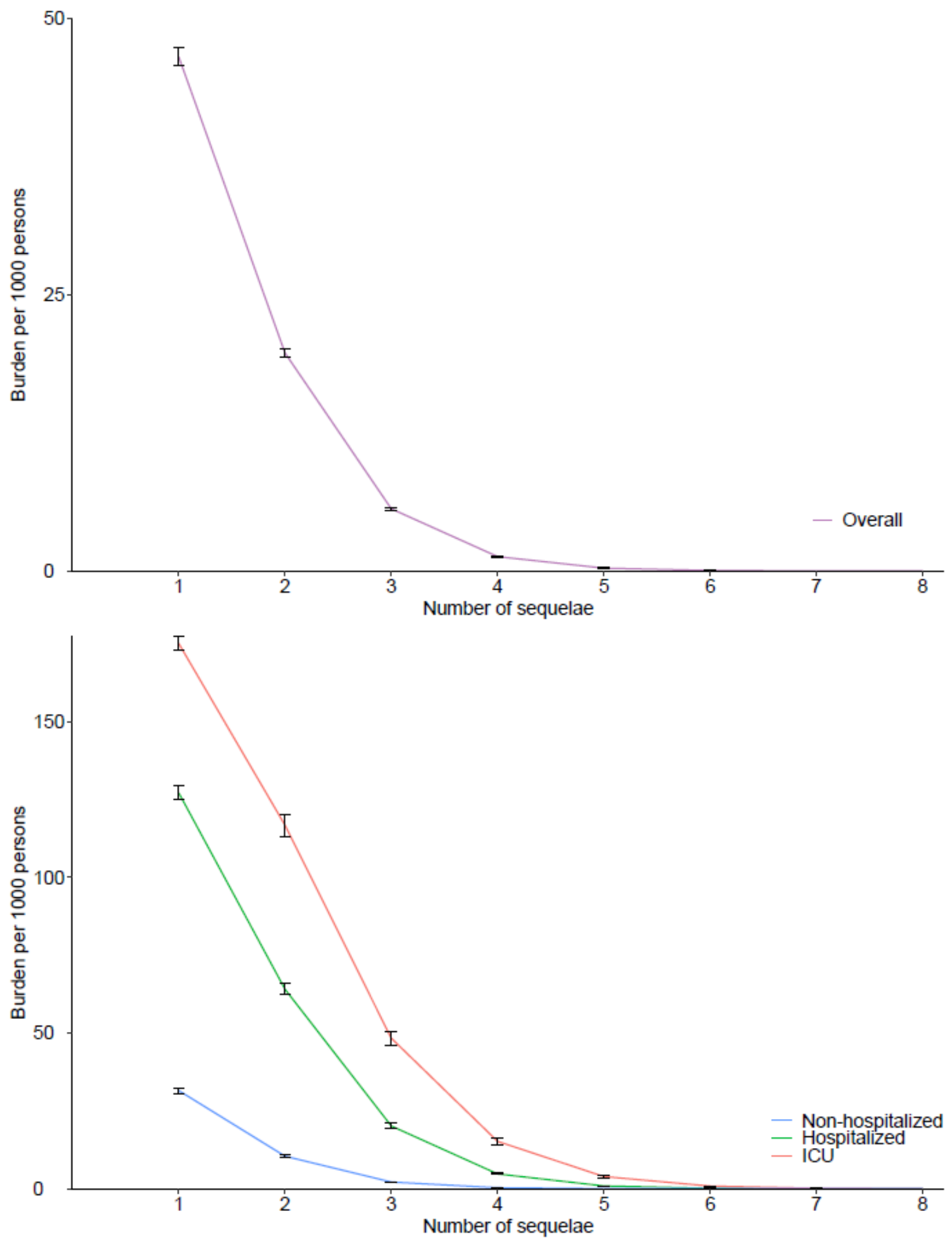

\section{Figure 1}

Burden of Post-acute Sequelae of COVID-19 as a function of the number of sequelae. a) overall cohort, and b) by care setting (non-hospitalized, hospitalized, and admitted to intensive care during the acute phase of the infection). Post-acute sequalae were ascertained from 30 days after infection until end of 
follow-up. Estimates of burdens per 1000 COVID-19 patients and 95\% confidence intervals at 6-months are presented.

\begin{tabular}{|c|c|c|c|c|c|c|}
\hline & Overall & $\begin{array}{l}\text { Age } \\
=50 \\
\end{array}$ & $\begin{array}{c}\text { Age } \\
60-70\end{array}$ & $\begin{array}{l}\text { Age } \\
>70\end{array}$ & Black & white \\
\hline $\begin{array}{c}\text { Shortness } \\
\text { of breath }\end{array}$ & 28.80 & 23.56 & 32.41 & 27.82 & 30.13 & 28.39 \\
\hline $\begin{array}{r}\text { Sleep } \\
\text { disorders }\end{array}$ & 19.51 & 25.07 & 19.51 & 19.38 & 17.32 & 20.48 \\
\hline Hyperlipidemla - & 17.09 & 21.85 & 21.25 & 13.32 & 15.23 & 17.64 \\
\hline $\begin{array}{c}\text { Chest } \\
\text { pain }\end{array}$ & 13.84 & 16.55 & 16.13 & 9.91 & 16.46 & 13.07 \\
\hline Fatigue- & 13.59 & 5.40 & 12.87 & 22.79 & 13.94 & 13.29 \\
\hline GERD- & 13.25 & 8.41 & 14.46 & 17.80 & 11.04 & 14.11 \\
\hline Thromboembollsm - & 11.35 & 5.65 & 12.55 & 15.66 & 13.32 & 10.64 \\
\hline Cough - & 10.24 & 9.87 & 13.37 & 8.13 & 12.37 & 9.37 \\
\hline $\begin{array}{r}\text { Muscle } \\
\text { wwakness }\end{array}$ & 9.81 & 2.54 & 8.45 & 19.48 & 10.89 & 9.38 \\
\hline $\begin{array}{l}\text { Joint } \\
\text { pain }\end{array}$ & 8.92 & 6.52 & 9.42 & 11.47 & 10.25 & 8.42 \\
\hline Anxlety & 8.75 & 5.04 & 11.03 & 12.33 & 9.83 & 8.52 \\
\hline Dlabetes mellitus - & 8.47 & 4.67 & 10.46 & 11.07 & 11.31 & 7.29 \\
\hline Arrythmlas - & 7.85 & 8.06 & 7.00 & 6.81 & 7.66 & 7.83 \\
\hline Depression - & 7.61 & 8.51 & 8.19 & 7.81 & 7.93 & 7.41 \\
\hline $\begin{array}{l}\text { Chronic } \\
\text { kidney disease }\end{array}$ & 7.19 & 0.87 & 6.53 & 19.24 & 6.63 & 7.52 \\
\hline Constipation - & 7.13 & 2.77 & 5.81 & 12.51 & 8.40 & 6.67 \\
\hline $\begin{array}{l}\text { Memory } \\
\text { problems }\end{array}$ & 6.46 & 1.91 & 4.25 & 14.63 & 5.93 & 6.86 \\
\hline Hypoxemla - & 6.43 & 2.72 & 6.89 & 10.25 & 5.92 & 6.84 \\
\hline $\begin{array}{r}\text { Acute coronary } \\
\text { disease }\end{array}$ & 6.18 & 1.12 & 6.84 & 15.07 & 5.63 & 6.45 \\
\hline $\begin{array}{r}\text { Acute } \\
\text { kidney injury }\end{array}$ & 6.07 & 1.33 & 6.36 & 12.32 & 9.15 & 5.08 \\
\hline Tachycardla & 4.69 & 4.04 & 5.32 & 4.69 & 5.75 & 4.29 \\
\hline $\begin{array}{c}\text { Dlabetes } \\
\text { mellitus }\end{array}$ & 4.68 & 4.62 & 4.42 & 4.78 & 5.15 & 4.62 \\
\hline $\begin{array}{l}\text { Mood } \\
\text { disorder }\end{array}$ & 4.57 & 6.79 & 4.06 & 4.78 & 5.58 & 4.04 \\
\hline Headache & 3.80 & 7.09 & 5.60 & 3.00 & 5.24 & 3.32 \\
\hline obestif & 3.49 & 5.57 & 5.11 & 2.67 & 3.09 & 3.78 \\
\hline $\begin{array}{l}\text { Skin } \\
\text { rash }\end{array}$ & 3.30 & 3.22 & 3.29 & 3.30 & 2.37 & 3.66 \\
\hline $\begin{array}{r}\text { Substance } \\
\text { abuse }\end{array}$ & 3.24 & 3.43 & 4.22 & 2.59 & 5.28 & 2.35 \\
\hline Stroke- & 3.11 & 0.83 & 3.24 & 5.44 & 3.51 & 2.94 \\
\hline Eradycardla - & 2.63 & 1.43 & 2.26 & 4.17 & 2.03 & 2.89 \\
\hline $\begin{array}{c}\text { Heart } \\
\text { fallure }\end{array}$ & 2.02 & 0.20 & 1.72 & 4.88 & 2.07 & 1.97 \\
\hline $\begin{array}{l}\text { Smell } \\
\text { problems }\end{array}$ & 1.34 & 2.09 & 1.15 & 0.80 & 0.88 & 1.42 \\
\hline Myocardits & 0.32 & 0.22 & 0.30 & 0.28 & 0.29 & 0.30 \\
\hline $\begin{array}{l}\text { Halr } \\
1055\end{array}$ & 0.22 & 0.70 & 0.27 & 0.06 & 0.10 & 0.29 \\
\hline
\end{tabular}

\begin{tabular}{|c|c|c|c|c|}
\hline Male & Female & $\begin{array}{c}\text { No } \\
\text { comorbiatles }\end{array}$ & $\begin{array}{l}1-3 \\
\text { comorolditis }\end{array}$ & $\begin{array}{c}>3 \\
\text { :omorbldilles }\end{array}$ \\
\hline 28.04 & 33.55 & 20.27 & 29.79 & 37.50 \\
\hline 20.31 & 15.22 & 16.73 & 18.39 & 25.51 \\
\hline 17.78 & 11.96 & 19.99 & 14.29 & 8.89 \\
\hline 13.49 & 18.01 & 11.16 & 13.44 & 12.75 \\
\hline 14.30 & 7.21 & 6.96 & 15.20 & 33.42 \\
\hline 14.16 & 6.67 & 8.96 & 13.71 & 22.75 \\
\hline 12.02 & 5.02 & 6.96 & 12.62 & 18.39 \\
\hline 10.04 & 13.29 & 8.57 & 9.11 & 9.77 \\
\hline 10.62 & 2.85 & 3.62 & 12.20 & 28.12 \\
\hline 9.65 & 3.30 & 5.60 & 9.65 & 15.75 \\
\hline 9.20 & 5.86 & 4.84 & 9.14 & 18.40 \\
\hline 9.18 & 3.37 & 8.11 & 7.98 & 10.59 \\
\hline 7.51 & 10.90 & 6.76 & 6.82 & 8.11 \\
\hline 7.83 & 7.25 & 4.98 & 7.98 & 12.77 \\
\hline 7.84 & 3.34 & 3.60 & 10.57 & 20.17 \\
\hline 7.29 & 6.01 & 3.31 & 8.44 & 14.74 \\
\hline 6.99 & 2.05 & 4.14 & 6.44 & 13.32 \\
\hline 6.89 & 2.39 & 3.03 & 7.69 & 15.81 \\
\hline 7.22 & 0.18 & 2.69 & 8.05 & 17.39 \\
\hline 6.92 & 1.30 & 2.62 & 8.87 & 25.07 \\
\hline 4.63 & 5.31 & 2.83 & 5.02 & 8.18 \\
\hline 4.76 & 4.62 & 2.94 & 4.44 & 8.41 \\
\hline 4.71 & 4.39 & 3.87 & 4.14 & 7.10 \\
\hline 3.50 & 11.44 & 3.74 & 3.45 & 4.14 \\
\hline 3.54 & 4.25 & 3.54 & 3.09 & 3.55 \\
\hline 3.05 & 5.77 & 2.70 & 3.39 & 2.56 \\
\hline 3.53 & 0.92 & 3.49 & 2.33 & 4.64 \\
\hline 3.31 & 1.46 & 1.32 & 3.69 & 6.53 \\
\hline 2.73 & 1.67 & 1.84 & 2.95 & 3.27 \\
\hline 2.27 & 0.33 & 0.81 & 2.80 & 9.12 \\
\hline 1.28 & 1.70 & 1.35 & 1.10 & 0.89 \\
\hline 0.34 & 0.00 & 0.11 & 0.20 & 0.57 \\
\hline 0.13 & 3.59 & 0.31 & 0.20 & 0.10 \\
\hline
\end{tabular}

Burden 0

$10 \quad 20$

30

40

\section{Figure 2}

Burden of Post-acute Sequelae of COVID-19 as a function of the number of sequelae. a) overall cohort, and b) by care setting (non-hospitalized, hospitalized, and admitted to intensive care during the acute phase of the infection). Post-acute sequalae were ascertained from 30 days after infection until end of 
follow-up. Estimates of burdens per 1000 COVID-19 patients and 95\% confidence intervals at 6-months are presented.
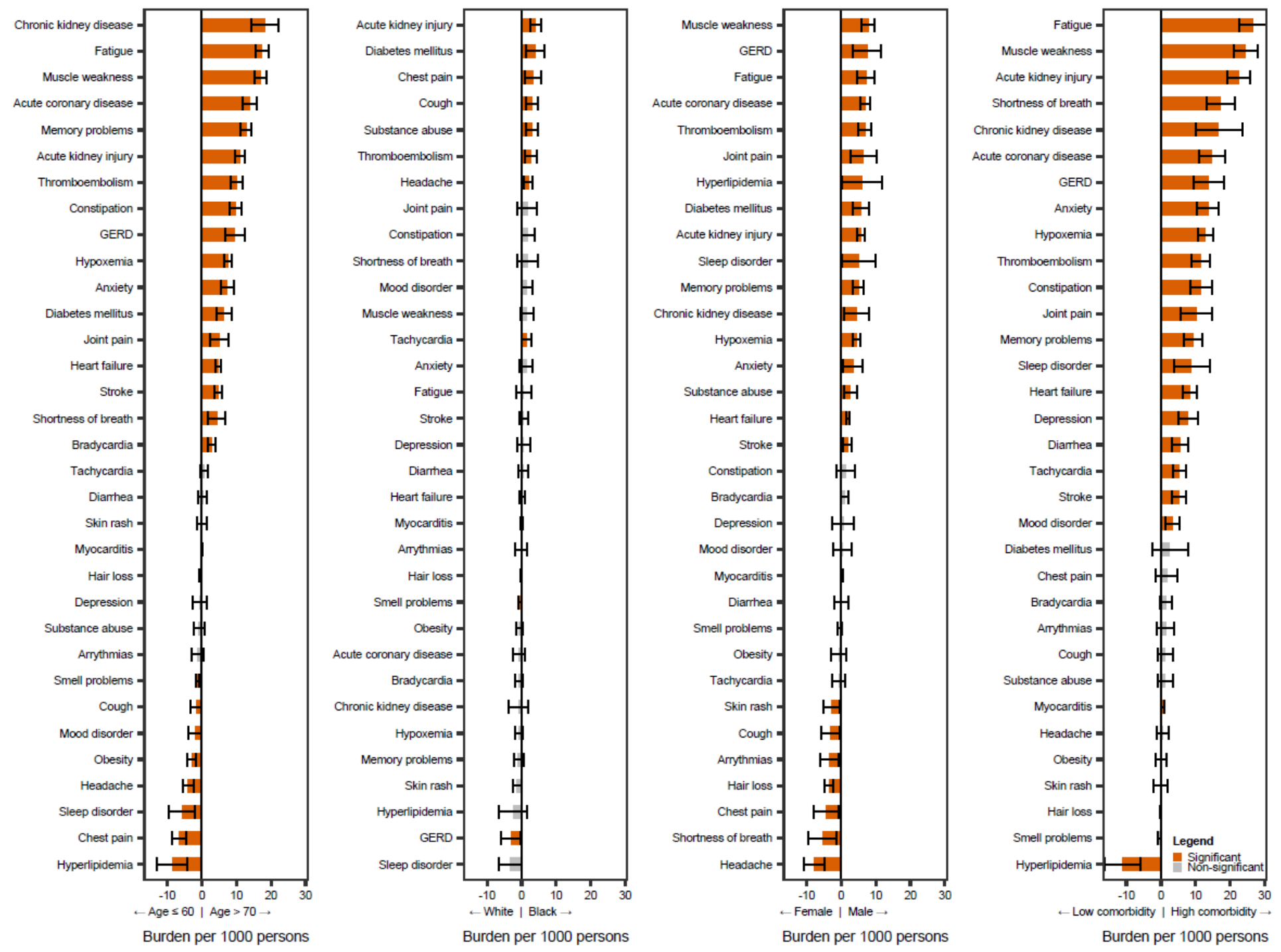

Figure 3

Differences in burden of individual Post-acute Sequelae of COVID-19 by age, race, sex, and health status. Differences in burden per 1000 COVID-19 patients and 95\% confidence intervals at 6-months are presented. 

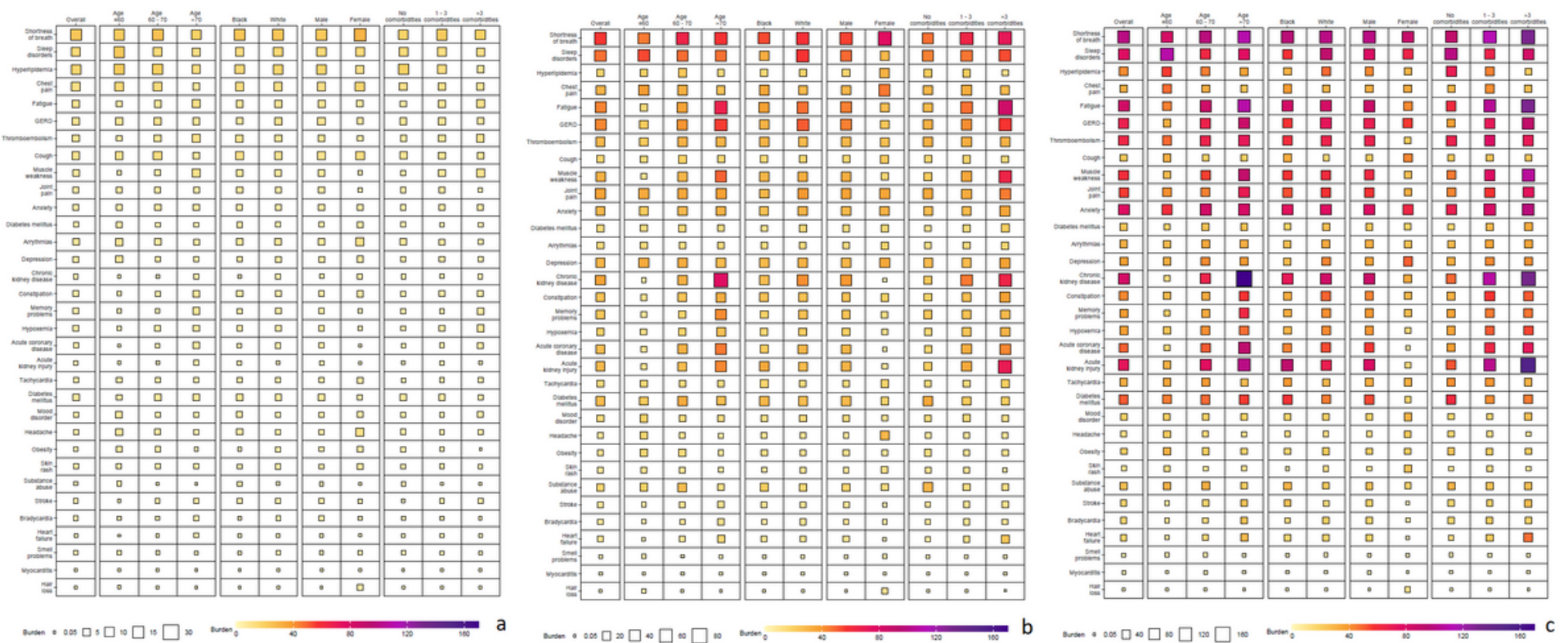

\section{Figure 4}

Burden of Post-acute Sequelae of COVID-19 in the overall cohort and by age, race, sex, and health status in each care setting. Burden of individual sequelae in non-hospitalized (a), hospitalized (b), and those admitted to intensive care (c). Estimates of burdens per 1000 COVID-19 patients at 6-months are presented. The size of the square represents the burden within each care setting. The intensity of color from light yellow to deep purple represents the range of burdens across care settings.

\section{Supplementary Files}

This is a list of supplementary files associated with this preprint. Click to download.

- Supplementaryinformation.docx 